\title{
The Derivation of the Cosmic Microwave Background Radiation Peak Spectral Radiance, Planck Time, and the Hubble Constant from the Neutron and Hydrogen
}

\author{
Donald William Chakeres ${ }^{1}$, Vola Andrianarijaona ${ }^{2}$ \\ ${ }^{1}$ Department of Radiology, The Ohio State University, Columbus, OH, USA \\ ${ }^{2}$ Department of Physics \& Engineering, Pacific Union College, Angwin, CA, USA \\ Email: donald.chakeres@osumc.edu, vola@puc.edu
}

Received 22 February 2016; accepted 28 March 2016; published 31 March 2016

Copyright ( 2016 by authors and Scientific Research Publishing Inc.

This work is licensed under the Creative Commons Attribution International License (CC BY).

http://creativecommons.org/licenses/by/4.0/

(c) (i) Open Access

\section{Abstract}

Purpose: The cosmic microwave background radiation, CMB, is fundamental to observational cosmology, and is believed to be a remnant from the Big Bang. The CMB, Planck time, $t_{p}$, and the Hubble constant, $H_{0}$, are important cosmologic constants. The goal is to accurately derive and demonstrate the inter-relationships of the CMB peak spectral radiance frequency, $t_{P}$, and $H_{0}$ from neutron and hydrogen quantum data only. Methods: The harmonic neutron hypothesis, $\mathrm{HNH}$, evaluates physical phenomena within a finite consecutive integer and exponential power law harmonic fraction series that are scaled by a fundamental frequency of the neutron as the exponent base. The CMB and the $H_{0}$ are derived from a previously published method used to derive $t_{P}$. Their associated integer exponents are respectively $+1 / 2,-3 / 4$, and $-128 / 35$. Results: Precise mathematical relationships of these three constants are demonstrated. All of the derived values are within their known observational values. The derived and known values are: $v_{C M B}, \mathbf{1 6 0 . 0 4 1 7 3 7}(06)$ $\times 10^{9} \mathrm{~Hz}, \sim 160 \times 10^{9} \mathrm{~Hz} ; 2.72519 \mathrm{~K}, 2.72548 \pm 0.00057 \mathrm{~K}, H_{0} 2.29726666(11) \times 10^{-18} \mathrm{~s}^{-1}, \sim 2.3 \times$ $10^{-18} \mathrm{~s}^{-1}$; and $t_{P} 5.3911418(3) \times 10^{-44} \mathrm{~s}, 5.39106(32) \times 10^{-44} \mathrm{~s}$. Conclusion: The cosmic fundamental constants $t_{P}, H_{0}$, and $C M B$ are mathematically inter-related constants all defined by gravity. They are also directly derivable from the quantum properties of the neutron and hydrogen within a harmonic power law.

\section{Keywords}

Cosmic Microwave Background Radiation, Neutron, Fundamental Physical Constants, Unification Models, Hydrogen, Planck Time, Hubble Constant 


\section{Introduction}

\subsection{The Cosmic Background Microwave Radiation}

The cosmic microwave background radiation, CMB, is fundamental to observational cosmology, and is believed to be a remnant of the thermal radiation from the Big Bang [1]-[13]. It is considered a landmark test of the Big Bang model of the Universe. There has recently developed questioning if this is the only possible interpretation [14]. Utilizing quantum methods perhaps a Big Bang singularity can be avoided. This perspective is not well established, and not the standard approach.

CMB measurements have been made with a series of probes including: NASA's Cosmic Background Explorer (COBE) satellite that orbited in 1989-1996, the ESA (European Space Agency) Planck Surveyor in 2009, and Planck cosmology probe all-sky map data from 2013. The experimental value of the CMB peak spectral radiance $v_{\max }$ is approximately $160 \mathrm{GHz}$. The most recent reported associated temperature is $2.72548 \pm 0.00057 \mathrm{~K}$. The CMB cannot be derived by the Standard Model(SM) or String Theory (ST) [15].

\subsection{Planck Time and the Hubble Constant}

Planck time, $t_{P}$, is an important unique unifying composite cosmology constant that interlinks the speed of light $c$, Planck's constant $h$, and the Newtonian gravitational constant $G$ that are classical, quantum, and cosmologic constants. The units of $t_{P}$ are seconds, inverse $\mathrm{Hz}$ [16] The Hubble constant, $H_{0}$, is also linked to the Big Bang and is felt to represent a measure of the expansion rate of the universe [17]-[22]. Though frequently quoted as a velocity, its actual unit is per second or Hz. The $H_{0}$ does not represent a fixed constant, but rather an evolving one that is only measured presently as a transient value. These two cosmology constants along CMB are defined by units of time either seconds or $\mathrm{Hz}$ so they can be directly inter-related and mathematically scaled as dimensionless ratios. These constants are presently not understood to represent an integrated system directly linking them into a coherent mathematical or physical system where one value can be used to derive another like many other physical systems, for example, the Bohr radius, $\mathrm{a}_{0}$.

\subsection{Paper Structure}

The structure of this paper follows a logical progression. An overview of the Harmonic Neutron Hypothesis, $\mathrm{HNH}$, is introduced including the concepts of a dimensionless ratio fundamental constant system, Buckingham Pi theory, a single natural unit system related to the neutron, and integer based power laws. The fundamental constants are converted to frequency equivalents then dimensionless ratios. These dimensionless constants are converted to harmonic partial fraction exponents of the frequency equivalent of the neutron. A review of the universal nature of Planck time within physics and how it is derived within the HNH are demonstrated. From the Planck time power law the Hubble constant is derived based on its partial harmonic fraction. The Hubble constant is evaluated as a $\beta$ velocity ratio. The kinetic energy associated with the Hubble constant $\beta$ is derived. The assignment of the harmonic fraction related to $\mathrm{CMB}$ is logically analyzed. Utilizing the identical kinetic energy pattern related to the Hubble constant $\beta$ the peak spectral radiance of CMB is derived. CMB is transformed to a Kelvin temperature using the Wien's displacement factor. Mathematical methods to transform Planck time to the Hubble constant or to CMB are shown. The derived values are compared to the experimental known values. A discussion of the insights into the fundamental constants as a classic harmonic system is made. Finally the conclusions are summarized.

\section{Goal}

The goal of this work is to derive and predict the peak spectral radiance, $v_{\max }, v_{\mathrm{CMB}}$ and the associated temperature using the Wien's displacement factor, $t_{P}$ and $H_{0}$ from the subatomic data of the frequency equivalents $v$ of the neutron $\left(n^{0}\right)$, electron $\left(e^{-}\right)$, Bohr radius $\mathrm{a}_{0}$, the Rydberg constant $R$, and integers. The methods of the HNH will be utilized [23]-[31]. The HNH has previously accurately derived $t_{P}, H_{0}$, Higgs boson, $H^{0}$, the quarks, and the energy lost in neutron beta decay from the same limited subatomic data and integer exponent fractions, Table 1. CMB's close mathematical relationships to the $H_{0}, t_{p}$, and the concept of fine structure $\beta$ constant, $\alpha$ will be demonstrated. 
Table 1. Values utilized in the derivation of $\mathrm{CMB}_{\mathrm{d}}$ and the Known Values. Table 1 lists the published values of vs, and the slopes and intercepts of the $w k$ and $e m$ lines used for this derivation of CMB. The known and derived values of $t_{P}^{2}, H_{0}$, and $\mathrm{CMB}$ are listed. The derivations fall within the known experimental ranges.

\begin{tabular}{|c|c|}
\hline physical constant $\left(\right.$ derived $_{d}$ and known $_{k}$ ) & value \\
\hline$\left[\left(v_{n^{0}}\right) s\right]$ & $2.2718590(01) \times 10^{23}$ \\
\hline $\log _{e}\left[\left(v_{n^{0}}\right) s\right]$ & $53.780055612(22)$ \\
\hline$b w k$ : y intercept, weak force, $w k$ line & $3.51638329(18) \times 10^{-3}$ \\
\hline$a w k$ : slope, weak force, $w k$ line & $3.00036428(15) \times 10^{-3}$ \\
\hline bem: y intercept, electromagnetic, em line & $-3.45168347(17) \times 10^{-3}$ \\
\hline aem: slope, electromagnetic, em line & $-3.45168347(17) \times 10^{-3}$ \\
\hline$t_{P d}^{2}$, no $\hbar$ & $1.82617124(09) \times 10^{-86} \mathrm{~s}^{2}$ \\
\hline$t_{P k}^{2}$, no $\hbar$ & $1.82611(11) \times 10^{-86} \mathrm{~s}^{2}$ \\
\hline$t_{P d}$, with $\hbar$ & $5.3911418(3) \times 10^{-44} \mathrm{~s}$ \\
\hline$t_{P k}$, with $\hbar$ & $5.39106(32) \times 10^{-44} \mathrm{~s}$ \\
\hline$H_{0 d}$ & $2.29726666(11) \times 10^{-18} \mathrm{~s}^{-1}$ \\
\hline$H_{0 k}$ & $\sim 2.3 \times 10^{-18} \mathrm{~s}^{-1}$ \\
\hline $\mathrm{CMB}_{\mathrm{d}}, v_{\max }$ & $160.041737(06) \times 10^{9} \mathrm{~Hz}$ \\
\hline $\mathrm{CMB}_{\mathrm{k}}, v_{\max }$ & $\sim 160 \times 10^{9} \mathrm{~Hz}$ \\
\hline derived CMB in Temperature & $2.72519 \mathrm{~K}$ \\
\hline known CMB in Temperature & $2.72548 \pm 0.00057 \mathrm{~K}$ \\
\hline
\end{tabular}

\section{An Overview of the Harmonic Neutron Hypothesis, $\mathrm{HNH}$}

The following is a limited review and explanation of the HNH. The details have been described in multiple previous publications, and will not be repeated [23]-[31]. Our model is purely computational and finite integer based so there are no singularities. The model is not based primarily on observations of physical systems, but defined by pure integer mathematical properties. The primary hypothesis is that the fundamental constants are inter-related by simple, ubiquitous integer mathematical patterns and properties. The model is a logical extension of Planck's postulate. That is the only possible quantum values that are related to a harmonic system based on standing wave mathematical properties. The model assumes the global physical system is an integrated harmonic one. The model is based on pure integer number properties of finite consecutive integer series as integers, $n$, harmonic fractions, $1 / n$, for $n$ as a consecutive integer series; and partial harmonic fractions, $1-(1 / n)$.

The whole system is scaled by a single natural unit fundamental frequency, $v_{f}$. In this model the annihilation frequency of the neutron, $n^{0}$, times by 1 second, $2.2718590(01) \times 10^{23} \mathrm{~Hz} \times s,\left[\left(v_{n^{0}}\right) s\right]$, is a dimensionless fundamental constant that is invariant by definition when evaluated within a single unit coupling constant system similar to Planck units. These integers and fractions define a global harmonic system that encompasses the geometry of a circle, and therefore all of the associated properties of waves including $\pi$, frequency, and phase. All harmonic systems are power laws and therefore exponential by definition, though most commonly viewed from the linear perspective. The pure number properties such as even, odd, prime, composites, squares, sums, square roots, etcetera dominate and explain the organization of the model and secondarily all physical systems [29].

The HNH is a single natural unit harmonic system spanning different physical domains. Each physical state, distance, time, matter are defined with an integer scaled by the fundamental frequency, $v_{F}$ in the linear domain. Each force/physical domain unit is defined by an integer exponent of $\left[\left(v_{n^{0}}\right) s\right]\{-1,0,1,2\}$. Exponent -1 is 
the unit for elemental gravity. This is related to two times the gravitational binding energy of the electron in hydrogen, $2 \times 2.9001 \times 10^{-24} \mathrm{~Hz}$. The reciprocal of $\left[\left(v_{n^{0}}\right) s\right]$ is $4.4017 \times 10^{-24}$ so they are nearly identical. This is equivalent to the ionization energy of hydrogen, but in the gravitational domain. The exponent 0 is associated with $1 \mathrm{~Hz}$ which is related to the Hz equivalent of Planck's constant, $h$. Each electromagnetic wave is in integer $\mathrm{Hz}$, the unit for electromagnetic energy. Exponent 1 is related to the neutron, and the unit for atoms. The exponent 2 is related to the next logical consecutive integer of the series. This is related to a mass equivalent of $\left[\left(v_{n^{0}}\right) s\right]$ neutrons or $\left[\left(v_{n^{0}}\right) s\right]^{2} \mathrm{~Hz}$. This is equivalent to $5.1613 \times 10^{46} \mathrm{~Hz}$ or $3.8052 \times 10^{-4} \mathrm{~kg}$. This mass must be a mass within a black hole state. The Compton wavelength of this dense mass is smaller than it Schwarzschild radius. Therefore, the exponent consecutive series corresponds to the units for gravity, electromagnetic, atomic, and black hole domains.

The frequency equivalent of other observable physical constants and particles, such as the Bohr radius, $\mathrm{a}_{0}$, the Rydberg constant, $R$, Higg's boson, $H^{0}$, the electron, $e^{-}$, leptons, bosons, quarks, mesons are also associated with an integer, $n$, in the exponential domain. This $n$ defines a partial fraction, $1 \pm(1 / n)$, that is an exponent of the $\left[\left(v_{n^{0}}\right) s\right]$. This integer is logically and computationally derived based on relative scale similar in concept to the chemical periodic chart and must also fall on defined power law lines.

The physical constants also follow power law properties within a harmonic system with the four natural unit integer values that scale the system, namely the $n^{0}, e^{-}, \mathrm{a}_{0}$, and $R$. Only two of the hydrogen natural units need to be known since the other can be derived from other two. Therefore there are three essential natural units. $\left[\left(v_{n^{0}}\right) s\right]$, the frequency equivalents of the $e^{-}, \mathrm{a}_{0}$, and $R$ scale the global system. The model has predictive power beyond standard methods by analyzing the system as an integrated harmonic spectrum rather than individual, isolated physical values. Harmonic systems have an inherent predictive power where if the $v_{F}$ is known then all of the possible harmonics can be defined or vice versa. This mathematical property accounts for the possibility of deriving cosmologic constants from non-cosmologic physical data (subatomic data in particular).

The model utilizes the dimensional analysis methods similar to that of Rayleigh and Buckingham's Pi Theorem. The Buckingham Pi Theorem states that physical laws are independent of the form of the physical units. Therefore, acceptable laws of physics are homogeneous in all dimensions.

All of the physical phenomena are evaluated as frequency equivalents, and secondarily as dimensionless coupling constant ratios. The system is physical unit-independent. The fundamental frequency, $v_{F}$, ratio we use as a dimensionless number, is that scaled by the annihilation frequency of the neutron. Most other units are 1 , and drop out. All entities are defined by exponents of $v_{F}$ for the constants or integer values for the individual states.

\section{Harmonic Neutron Hypothesis Previous Derivations of Fundamental Constants from Subatomic Data only}

All predictions and derivation are from two (2) finite integer sets. The first set includes four natural units based on known atomic quantities as frequency equivalents. Included in this set is the neutron, $n^{0}$, and comprises $v_{F}$. A partition of this set includes the frequency equivalents of the $v_{e^{-}}, v_{a_{0}}$, and $v_{R}$. From this initial data composite universal slopes and Y-intercepts of two lines have been reported, Figure 1. These slopes and Y-intercepts are $a w k, b w k$, and aem, bem; and refer to weak kinetic properties, $w k$, and electromagnetic ones, em, Table 1 . This is the only physical data for all of the derivations. It is hypothesized that all of the fundamental constants can be derived from this limited data set. Other fundamental constants are related to these values in a logical fashion. The second set is related to the integer fractions. In this paper the $n$ include: $\{1,2,3,4,5,6,7,35,128\}$.

The unit system of the HNH is simplified with the units for Planck's constant, electric charge, time, the distance light travels in one unit of time, and the speed of light all equaling 1 . All entities are defined as in terms of ratios of two linear states, or exponents of $v_{F}$. Energy, mass, frequency, temperature are all scaled equally. Distance is defined as 1 divided by frequency, $1 / v$. The speed of light equals $\left[\left(v_{n^{0}}\right) s\right]$ times the distance $1 /\left[\left(v_{n^{0}}\right) s\right]$, the Compton wavelength of the neutron. 


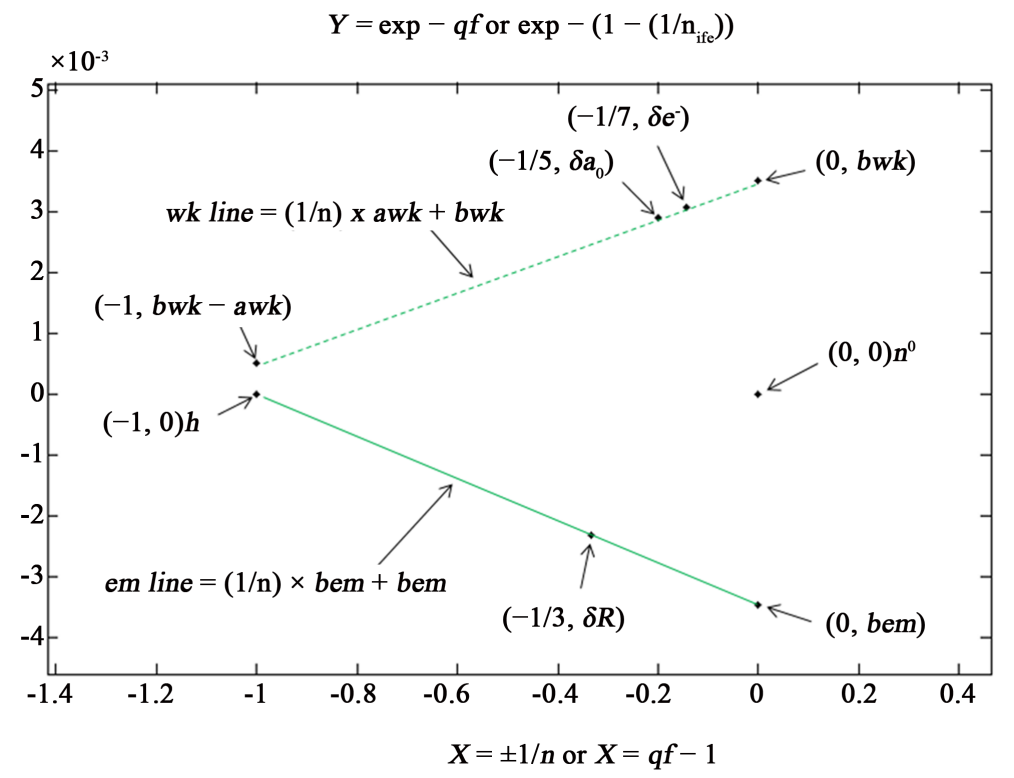

Figure 1. Power Law 2D Plot Neutron and Hydrogen Quantum Values. Figure 1 is a 2D power law plot. The $\mathrm{X}$-axis equals the $q f \mathrm{~s}$ minus 1 . The $\mathrm{Y}$-axis is the difference, $\delta$, between the known or derived exponents and their qfs. The previously published points related to $h, n^{0}, e^{-}, R, \mathrm{a}_{0}$ are plotted. The X-axis is scaled by the neutron and $h$. The $w k$ line is associated with the points for $e$ and $\mathrm{a}_{0}$, dashed green line. The em line is associated with the points for $h$ and $R$, solid green line. These slopes and Y-intercepts are used in the derivation methods, Figure 2 and Figure 3.

\section{Methods and Results}

\subsection{Nomenclature and Abbreviations}

There are many fundamental constants and calculations utilizing these constants. For clarity they will be listed here. They include: neutron, $n^{0}, v$ refers to the frequency equivalent of a constant, electron, $e$-, Bohr radius, $\mathrm{a}_{0}$, Rydberg constant, $R$, speed of light, $c$, Planck's constant, $h$, Planck's time, $t_{P}$, Planck time squared, $t_{P}^{2}$, Hubble constant, $H_{0}$, Higgs boson, $H^{0}$, cosmic microwave background peak spectral radiance, CMB, $\beta$, a ratio of a velocity divided by $c, \alpha$, the fine structure constant, $\beta_{H_{0} d}$ the $\beta$ related to the Hubble constant, a $q f$ is an integer fraction exponent, and $\delta$ is the difference between a fundamental constant's exponent and its quantum fraction.

\subsection{Conversion of Physical Constants to Frequency Equivalents, Exponents, $\delta$, and Partial Fractions}

The known values are labeled with a subscript $k$, and the derived values with a subscript $d$. There are multiple possible integer "n's" described in this model and they are differentiated. Integer exponents, i.e., of $v_{F}$ are described as " $\mathrm{n}_{\mathrm{i}}$ ". Partial or harmonic integer fractional exponents, ife, " $\mathrm{n}$ " values are described as " $\mathrm{n}_{\mathrm{ife}}$ ", whereas the " $\mathrm{n}$ " of non-exponent linear entities of a consecutive integer series are described as " $\mathrm{n}_{\text {cis }}$ ". For example black body radiation energy equals $n_{\text {cis }}(v \mathrm{vh})$.

All of the data for the fundamental constants were obtained from http://physics.nist.gov/cuu/Constants/ and www.wikipedia.org. The NIST site http://physics.nist.gov/cuu/Constants/energy.html has an online physical unit converter that can be used for these types of calculations so the values used in the model are all standard unit conversions.

The floating point (the number of accurate digits) is based upon known experimental atomic data, of approximately $5 \times 10^{-8}$. Other constants have lower know accurate digits. All of the fundamental constants are converted to frequency equivalents, $v$. Equation (1) demonstrates the frequency equivalent conversion of the neutron as an example. $c$ is the speed of light, $h$ is Planck's constant, $m_{n^{0}}$ is the mass of the neutron. Though not intuitively obvious in this type of a single physical unit system, $v_{F}$, is an invariant coupling constant independent of whatever physical unit system is initially chosen. 


$$
v_{n^{0}}=\frac{c^{2}}{h} m_{n^{0}}=2.2718590(01) \times 10^{23} \mathrm{~Hz}
$$

Conversation of the other constants has been previously published. Masses are converted by multiplying by $c^{2}$ (speed of light squared) then dividing by $h$ (Planck's constant). Distances are converted by dividing the wavelength into $c$. Energies in Joules are converted to $\mathrm{Hz}$ by dividing by $h$. The $\mathrm{eV}$ value for the neutron is $939.565378(21) \times 10^{6}$. Its frequency in $\mathrm{Hz}$ is converted to $\mathrm{eV}$ by multiplying by the constant, $4.13566750(21) \times$ $10^{-15} \mathrm{eV} / \mathrm{Hz}$. The eV was converted to frequency by multiplying by the constant $2.41798930(13) \times 10^{14} \mathrm{~Hz} / \mathrm{eV}$.

This model has two parallel domains both describing identical physical values. One domain is the frequency equivalent of any physical value. The other domain is the exponent of the base $\left[\left(v_{n^{0}}\right) s\right]$ which when raised to that exponent equals the frequency equivalent of that specific value, Equation (2). Table 1 and Table 2 lists the standard unit quantum fractions, $\delta$ s, and exponents, exp of the constants evaluated in this paper. The exponent, exp, of a fundamental constant is the ratio of the $\log _{e}$ of the frequency equivalent, vs, divided by the $\log _{e}\left[\left(v_{n^{0}}\right) s\right]$, Equation (2). Here, $\log _{e}\left[\left(v_{n^{0}}\right) s\right]$ equals 53.780055612(22).

$$
\log _{v_{n^{0}} s}(v s)=\frac{\log _{e}(v)}{\log _{e}\left(v_{n^{0}} s\right)}=1 \pm \frac{1}{n_{i f e}}+\delta=q f+\delta
$$

The $\exp _{v_{0} s}$ minus the quantum fraction, $q f$, or partial harmonic fraction equals the $\delta$, and the Y-axis value when plotted, $y$, Equation (3). A quantum fraction, $q f$, is a possible integer fractional exponent, not solely a harmonic or partial fraction in some settings, for example composite constants like $t_{P}^{2}$. The frequency equivalent of a constant, $v$, is calculated by raising $\left[\left(v_{n^{0}}\right) s\right]$ to the $\exp$ in Equation (4). When the harmonic fraction is negative the entity has a mass less than that of the neutron, example, the electron, $-1 / 7$. When the harmonic fraction is positive the entity has a mass more than that of the $n^{0}$, example, $H^{0}, 1 / 11$.

or

$$
y=\delta=\exp -q f=\exp -\left(1 \pm \frac{1}{n_{\text {ife }}}\right)
$$

$$
\begin{gathered}
\exp =\left(1 \pm \frac{1}{n_{i f e}}\right)+y=\left(1 \pm \frac{1}{n_{\text {ife }}}\right)+\delta=q f+\delta=\Sigma q f+\Sigma \delta \\
v S=\left(v_{n^{0}} s\right)^{\left(1 \pm \frac{1}{n_{\text {ife }}}\right)+y}=\left(v_{n^{0}} s\right)^{\left(1 \pm \frac{1}{n_{\text {ife }}}\right)+\delta}=\left(v_{n^{0}}\right)^{q f+\delta}=\left(v_{n^{n}} s\right)^{\Sigma q f+\Sigma \delta}
\end{gathered}
$$

\subsection{The Power Law Two-Dimensional Plane}

Each physical entity is plotted on a log log plane in a power law format. The X-axis is related to the known or derived $q f$ or partial fraction minus 1, Figures 1-3. This centers the neutron at the $(0,0)$ central point. Each

\begin{tabular}{cccc}
\hline Table 2. List of the physical constant, $q f, \delta_{d}$, and $\exp _{d}$ of $t_{P d}^{2}, H_{0 d}, \beta_{H 0 d}, \quad v_{K E \beta H_{0 d}}$, and $v_{\mathrm{CMBd}}$. & \\
\hline Physical constant & $q f$ & $\delta$ & $-3.670879366(1)$ \\
\hline$t_{P d}^{2}$ no $\hbar$ & $-128 / 35$ & $-1.3736509(1) \times 10^{-2}$ & $-7.75520211(04) \times 10^{-1}$ \\
$H_{0 d}$ & $-3 / 4$ & $-5.20211263(26) \times 10^{-3}$ & $-7.5220175 \times 10^{-1}$ \\
$\beta_{H O d}$ & $-3 / 4$ & $-2.2017483 \times 10^{-3}$ & $-1.52029241(08)$ \\
$v_{K E \beta H_{0 d}}$ & $-3 / 2$ & $-2.02924155(10) \times 10^{-2}$ & $4.79707584(24) \times 10^{-1}$ \\
$v_{C M B d}$ & $1 / 2$ & $-2.02924155(10) \times 10^{-2}$ & \\
\hline
\end{tabular}




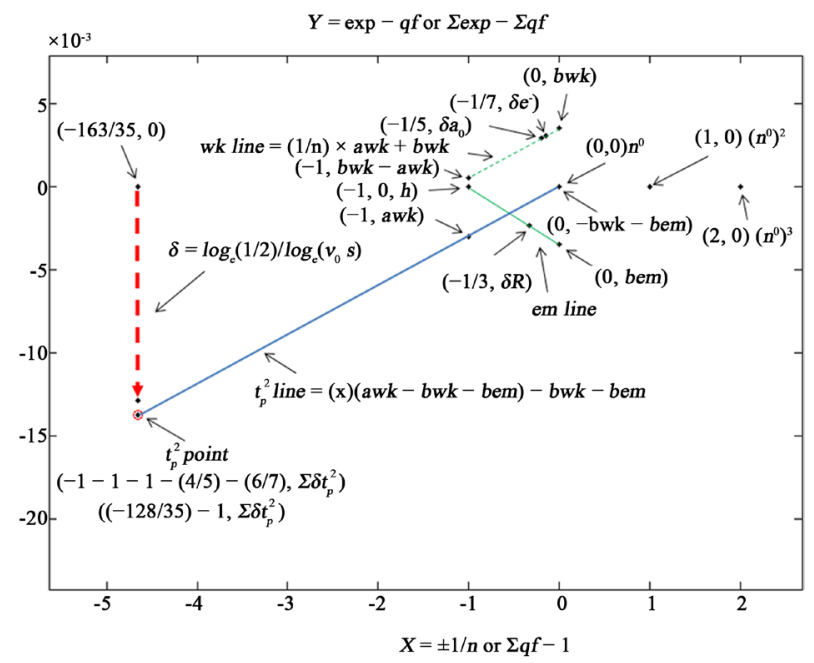

Figure 2. 2D power law 2D plot of known and derived $t_{p}^{2}$. Figure 2 is a power law plot. The $\mathrm{X}$-axis equals the qfs minus 1 . The Y-axis is the difference, $\delta$, between the known or derived exponents and their $q f \mathrm{~s}$. The previously published points related to $h, n^{0}, e^{-}, R, \mathrm{a}_{0}$ are plotted. The blue solid line is the $t_{P}^{2} \delta$-line. Planck time squared is plotted at X-axis [(-1-1$(4 / 5)-(6 / 7)) / 35)-1,(-128 / 35)-1,-163 / 35$. The $t_{p}^{2} \delta$-line traverses the points $(0,-b w k-b e m)$ and $(-1,-a w k)$. The red dashed arrow represents the vector value for $\log _{e}(1 / 2) / \log _{e}\left[\left(v_{n^{0}}\right) s\right]$ and extends from the $(-163 / 35,0)$ point down. The $t_{P}^{2} \delta$ is slightly smaller.

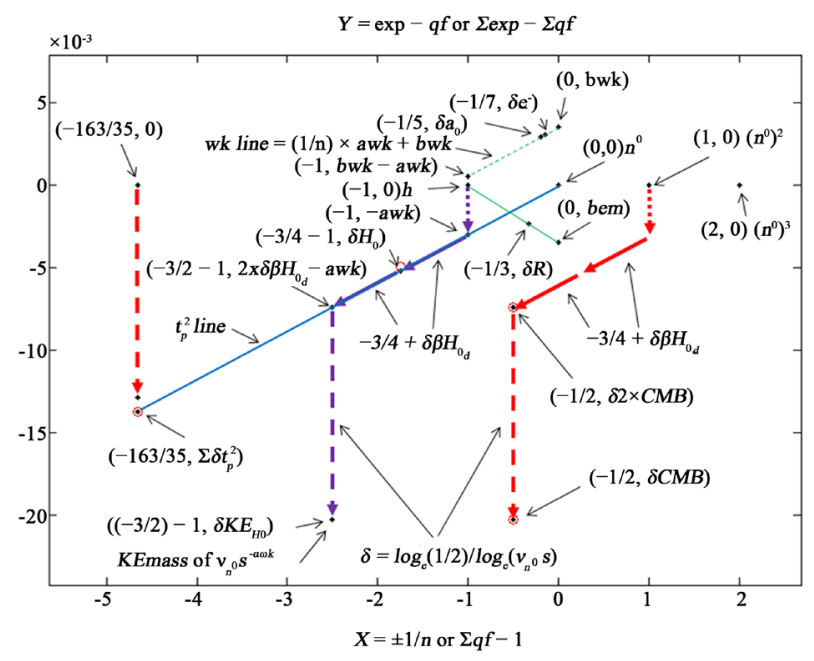

Figure 3. Power law plot of known and derived $t_{p}^{2}, H_{0}$, and CMB. Figure 3 is a 2D power law plot. The X-axis equals the qfs minus 1 . The Y-axis is the difference, $\delta$, between the known or derived exponents and their qfs. The previously published points related to $h, n^{0}, e^{-}, R, \mathrm{a}_{0}$ are plotted. The blue solid line is the $t_{P}^{2} \delta$-line. Planck time squared is plotted at $\mathrm{X}$-axis $-163 / 35$. The derived $H_{0}$ value, a black dot, is plotted at X-axis $(-3 / 4)-1$ on the $t_{P}^{2} \delta$-line. The known value is the red circle. The $K E_{H_{0}}$ point is plotted at $\mathrm{X}$-axis $(-3 / 2)-1$. The vertical dashed red and purple arrows equals $\log _{e}(1 / 2) / \log _{e}\left[\left(v_{n^{0}}\right) s\right]$. This is equivalent to dividing by 2 . The solid purple and red arrows are related to $\beta_{H_{0} d}$. The purple and red dotted arrows are related to $\left[\left(v_{n^{0}}\right) s\right]^{-a w k}$. The purple arrows are related to the derivation of the $K E_{H_{0}}$ and the red arrows are related to the derivation of CMB. The derivation of $K E_{H_{0}}$ begins at point $(-1,0)$ while the derivation of $\mathrm{CMB}$ begins at point $(1,0)$. The black dot is the derived $\mathrm{CMB}_{\mathrm{d}}$, and the known $\mathrm{CMB}_{\mathrm{k}}$ is the red circle plotted at $\mathrm{X}$-axis $(-1 / 2)-1$. 
integer division of the X-axis is an integer exponent change of $\left[\left(v_{n^{0}}\right) s\right] \cdot \log _{e}\left[\left(v_{n^{0}}\right) s\right]$ scales the X-axis. The $\mathrm{Y}$-axis is $\delta$, or $y$. The Y-axis is scaled by the properties of hydrogen and the composite slopes and Y-intercepts of $a w k, b w k$, and bem. Their derivation and origins are described in References [23] [27] [28], Table 1 and Table 2.

\subsection{Review of Previous Derivation Method for $t_{P d}^{2}$}

The classic $t_{P}$ equation when transformed to a frequency equivalent system relates the gravitational binding energy, GBE, as a frequency related to the product of Planck time squared, $t_{p}^{2}$ no $\hbar$; and the frequency equivalents of one mass, the other mass, and the distance separating them, Equation (5). $t_{P}^{2}$ equals the ratio of the frequency equivalent of the GBE divided by the product of the frequency equivalents of one mass, the other mass, and the distance separating them, Equation (6).

$$
\begin{aligned}
v G B E & =t_{P}^{2}\left(v_{m 1}\right)\left(v_{m 2}\right)\left(v_{\lambda}\right) \\
t_{P}^{2} & =\frac{v G B E}{\left(v_{m 1}\right)\left(v_{m 2}\right)\left(v_{\lambda}\right)}
\end{aligned}
$$

Planck time squared in the frequency domain is equivalent to the Newtonian gravitational constant, $G$. The sum of harmonic fractions defining $t_{p}^{2}$ is related to the gravitational binding energy of the electron in hydrogen include: $-1-1-(6 / 7)-(4 / 5)$, or $-128 / 35$. These are the fractions respectively associated with the proton, -1 , the gravitational binding energy of the electron in hydrogen, -1 , the $e^{-},-6 / 7$, and the $\mathrm{a}_{0},-4 / 5$.

The HNH has previously derived $t_{P d}^{2}, h$ not $\hbar$, Figure 2, Table 1 and Table 2 [25]. The line defining the $t_{P d}^{2}$ point is related to a composite sum of $a w k, b w k$, and bem just a $t_{P}$ is composite of three different important fundamental constants constants. The $\delta$ and exponent calculations are shown in Equations (7) and (8). The $\delta_{d}$, -1.3736509 (1) $\times 10^{-2}, \exp _{d},-3.670879366$ (1). The actual $t_{P d}^{2}$ is derived in Equation (9). The known experimental $t_{P k}^{2}$ value is $1.82611(11) \times 10^{-86} \mathrm{~s}^{2}$. The derived value is $1.82617124(09) \times 10^{-86} \mathrm{~s}^{2}$. The derived standard $\hbar$ Planck time is $5.3911418(3) \times 10^{-44} \mathrm{~s}$. The known experimental value is $5.39106(32) \times 10^{-44} \mathrm{~s}$.

$$
\begin{aligned}
& \delta_{t_{P d}^{2}}=-a w k+(-128 / 35) \times(a w k-b w k-b e m)=-1.3736509(1) \times 10^{-2} \\
& \exp _{t_{P_{d}}^{2}}=-128 / 35-a w k+(-128 / 35) \times(a w k-b w k-b e m)=-3.670879366(1) \\
& t_{P d}^{2}=\left[v_{n^{0}} s\right]^{(-128 / 35) \times(1+a w k-b w k-b e m)-a w k}=\left[v_{n^{0}} s\right]^{\exp _{t P^{2} d}=1.82617124(09) \times 10^{-86} \mathrm{~s}^{2}}
\end{aligned}
$$

\subsection{Review of Previous Derivation Method of $H_{0}$}

It was assumed that $H_{0 d}$ was associated with the $t_{p}^{2} \delta$-line since it is related to gravity, and a cosmic constant, Figure 2 and Figure 3 [28]. $H_{0}$ represents a reciprocal Lorentz factor, a red shift factor. Physically associated entities fall on a common power law line as seen with the quarks [26] [29] [31]. The $\mathrm{H}_{0}$ point falls on the $t_{p}^{2}$ line. $H_{0}$ is associated with the partial fraction of $-3 / 4$. The partial harmonic fraction $3 / 4$ is associated with the kinetic energy lost in the beta decay process [24]. On the power law the $H_{0}$ point is on the $t_{P}^{2}$ line at the $\mathrm{x}$ axis position $-3 / 4-1$ or $-7 / 4$. The previous derivation will not be repeated, but the methods are show in Equations (10) and (11), Table 1 and Table 2. The $\delta_{d},-5.20211263(26) \times 10^{-3}$, $\exp _{d},-7.75520211(04) \times 10^{-1}$, and actual $H_{0}$ are derived in Equation (12). The derived value is 2.29726666(11) $\times 10^{-18} \mathrm{~s}^{-1}$. This is within the known experimental range of approximately $2.3 \times 10^{-18} \mathrm{~s}^{-1}$. The WMAP data combined with other cosmological data generates the best $H_{0}$ estimate of $70.4 \pm 1.4(\mathrm{~km} / \mathrm{sec}) / \mathrm{Mpc}$. The derived value is $70.886246(4)(\mathrm{km} / \mathrm{sec}) / \mathrm{Mpc}$.

$$
\begin{gathered}
\delta_{H_{0 d}}=(-a w k-(3 / 4) \times(a w k-b w k-b e m))=-5.20211263(26) \times 10^{-3} \\
\exp _{H_{0 d}}=-a w k-(3 / 4) \times(1+a w k-b w k-b e m)=-7.55202110(4) \times 10^{-1} \\
H_{0_{d}}=\left(v_{n^{0}} s\right)^{(-a w k-(3 / 4) \times(1+a w k-b w k-b e m))}=2.2972666(11) \times 10^{-18} \mathrm{~s}^{-1}
\end{gathered}
$$




\subsection{The HNH $\beta$ and $\beta^{2}$ Properties and Power Law Plane}

In the HNH the ratio values of the points from $(-1,0)$ to $(-2,0)$ on the power law plane are equivalent to velocity ratios of $v$ divided by $c, v / c$, or a classic $\beta$ values utilized in Lorentz factor, red shift factor, time dilation factor of cosmology and high energy physics, Figure 2 and Figure 3. The X-axis has multiple different interpretations depending on the physical setting [27] [28]. In the HNH when the velocity/c equals 1 the $\beta$ is 1 . This is equivalent to the point $(-1,0)$. This is also the point for $\mathrm{h}$ and a frequency of $1 \mathrm{~Hz}$. All other velocities are fractional $\beta$ values. The $\beta$ values range from $1 /\left[\left(v_{n^{0}}\right) s\right]$ at $(-2,0)$ to 1 at $(-1,0)$.

In the HNH the ratio values of the points from $(-1,0)$ to $(-3,0)$ on the power law are related to velocity squared ratios of $v^{2}$ divided by $c^{2}$, or $\beta^{2}$ values, Figure 2 and Figure 3 . These $\beta^{2}$ range from $1 /\left[\left(v_{n^{0}}\right) s\right]^{2}$ at $(-3$, 0) to $1-\left(1 /\left[\left(v_{n^{0}}\right) s\right]^{2}\right)$ at $(-1,0)$.

\subsection{Conceptual Construct of the Fine Structure as a Specific $\beta$ Constant and a Virtual Kinetic Energy Transformation and Its Relationship to $\boldsymbol{H}_{0}$}

The original conceptual construct of the fine structure constant, $\alpha$, was related to the velocity ratio, $\beta$, of $v / c$ that would transform the energy equivalent of an electron going the speed of light, the annihilation energy, to the ionization energy of the electron. The $\alpha$ represents a specific $\beta$. This is a transformation of matter into either electromagnetic energy to kinetic energy. This velocity equals $\alpha \times c$ in standard units. The energy equivalent of the mass of an electron times $(c \alpha)^{2}$ divided by 2 is the ionization energy of hydrogen. An electron is not physically possible to have a velocity of $c$, but these types of "virtual" transformations are valid concepts in standard physics, and the HNH. This type of $\alpha$ virtual transformation is used multiple times in the derivation of CMB. All of the transformation on the power law plane are virtual, but remain mathematically, physically, and conceptually valid as well.

On the power law plane a $\beta$ represents a negative value vector. Its square is equal to two times that vector's distance. Any standard kinetic energy calculation can be made on the power law plane by starting at any mass equivalent then moving down or to the left twice the distance of the $\log _{e}(\beta) / \log _{e}\left(v_{n^{0}} s\right)$ distance then down or to the left the vector related to $\log _{e}(1 / 2) / \log _{e}\left[\left(v_{n^{0}}\right) s\right]$.

\subsection{Assignment of a Partial Harmonic Fraction $1 / 2$ to $v_{\mathrm{CMB}}$}

It is logical to associate CMB with the partial harmonic fraction of $1 / 2$. It has an even numbered denominator which is associated with kinetic properties [24]. It represents the lowest possible energy state of the positive partial fractions. CMB is logically associated as the minimum base kinetic energy state, and the smallest prime number, 2.

\subsection{Derivation of $\beta_{H_{0}}$ from $H_{0}$}

It is logical to analyze $H_{0}$ as associated with a specific $\beta_{H 0 d}$ identical to any kinetic process. This $\beta_{H_{0} d}$ represents the vector along the $t_{P}^{2}$ line starting from the virtual mass equivalent of the $\left[\left(v_{n^{0}}\right) s\right]^{-a w k}$ point, $0.8509858(42) \mathrm{Hz}$ at $\mathrm{X}$-axis value of -1 . It extends along the $t_{P}^{2}$ line to an $\mathrm{X}$-axis value of $-1-3 / 4$, or $-7 / 4$, the $H_{0_{d}}$ point. Here $\left[\left(v_{n^{0}}\right) s\right]^{a w k}$ equals 1.175107647. The $H_{0_{d}}$ is associated with a $\beta_{H_{0} d}$ of $\left[\left(v_{n^{0}}\right) s\right]^{(-0.775520211+a w k)}$, or, $H_{0_{d}} \times\left[\left(v_{n^{0}}\right) s\right]^{a w k}$, or 2.69953563(14) $\times 10^{-18}$, Figure 3, Equation (13), Table 1 and Table 2. The exponent of $\beta_{H_{0} d}$ equals $(-3 / 4) \times(1+a w k-b w k-b e m),-7.5220175 \times 10^{-1}$. The $\delta_{d}$ of $\beta_{H_{0} d}$ equals $(-3 / 4) \times(a w k-b w k-b e m),-2.2017483 \times 10^{-3} .\left(\beta_{H_{0} d}\right)^{2}$ equals $7.28749189(37) \times 10^{-36}$. 


$$
\beta_{H_{0_{d}}}=H_{0_{d}} \times\left(v_{n^{0}} s\right)^{a w k}=\left(v_{n^{0}} S\right)^{((-3 / 4) \times(1+a w k-b w k-b e m))}=2.69953563(14) \times 10^{-18}
$$

In standard experimental setting this $\beta_{H_{0} d}$ would be unmeasurable, but in the HNH these minute values are valid and essential. This equals $6.887032 \times 10^{-10} \mathrm{~m} / \mathrm{s}$ in standard units. $\left(\beta_{H_{0} d}\right)^{2}$ equals $4.743121 \times 10^{-19} \mathrm{~m}^{2} / \mathrm{s}^{2}$ in standard units.

\subsection{Derivation of the Kinetic Energy Related to $\beta_{H 0 d}$ and a Virtual Mass of $\left[\left(v_{n^{0}}\right) s\right]^{-a w k}$}

The kinetic energy, KE, frequency equivalent of the $H_{0 d}$ system, $v_{K E_{H_{0}} d}$, is related to the energy equivalent of a virtual mass of $\left[\left(v_{n^{0}}\right) s\right]^{-a w k}, 8.509858(42) \times 10^{-1} \mathrm{~Hz}$ times $H_{0}^{2}, 7.28749189(37) \times 10^{-36}$ divided by the product of 2 and $\left[\left(v_{n^{0}}\right) s\right]^{a w k}$. This equals $3.10077661(24) \times 10^{-36} \mathrm{~Hz}$, Figure 3, Equation (14) and Equation (15), Table 1 and Table 2. This represents the kinetic energy of a virtual mass equivalent slightly less than a frequency of 1 with a $\beta_{H_{0} d}$. Here $\log _{e}(1 / 2) / \log _{e}\left[\left(v_{n^{0}}\right) s\right]$ equals $-1.288855455 \times 10^{-2}$. The $v_{K E_{H_{0}} d}$ exponent equals $(-a w k-3 / 2 \times(1+a w k-b w k-b e m))$ plus $\log _{e}(1 / 2) / \log _{e}\left[\left(v_{n^{0}}\right) s\right],-1.50740386(08)-1.28885545 \times$ $10^{-2}$, or $-1.52029241(08)$. The partial fraction equals $-3 / 2$. The $\delta_{d}$ on the $t_{P}^{2}$ line equals

$(-a w k-3 / 2 \times(a w k-b w k-b e m)),-7.40386098(37) \times 10^{-3}$. The $\delta_{d}$ related to the actual energy equals $(-a w k-3 / 2 \times(a w k-b w k-b e m))$ plus $\log _{e}(1 / 2) / \log _{e}\left[\left(v_{n^{0}}\right) s\right]$, or $-2.02924155(10) \times 10^{-2}$. This is plotted on the $2 \mathrm{D}$ power law at the point $\left((-3 / 2-1),-2.02924155(10) \times 10^{-2}\right.$.

$$
\begin{gathered}
v_{K E_{H_{0_{d}}}}=\frac{\left.\left(v_{n^{0}} s\right)^{\left[-a w k-\frac{3}{2} \times(1+a w k-b w k-b e m)\right.}\right]}{2}=3.10077661(24) \times 10^{-36} \mathrm{~Hz} \\
v_{K E_{H_{0}}}=\frac{\left(H_{0 d}\right)^{2} \times\left(v_{n^{0}} s\right)^{a w k}}{2}=\frac{\beta_{H_{0_{d}}}^{2}}{2\left(v_{n^{0}} s\right)^{a w k}}=3.10077661(24) \times 10^{-36} \mathrm{~Hz}
\end{gathered}
$$

\subsection{Derivation of CMB from $t_{P d}^{2}$, the Kinetic Energy of $\boldsymbol{H}_{0 d}$, and $\left[\left(v_{n^{0}}\right) s\right]^{2}$}

The kinetic energy associated with $\beta_{H_{0} d}$ is related to a system identical to $\alpha$ and independent of any specific mass. The total ratio change from a frequency of 1 to $v_{K E_{H_{0}}}$ frequency is a constant, $3.10077661(24) \times 10^{-36}$. This transition should scale CMB as well since they are both related to gravity, are kinetic, and scaled by $H_{0}$, and $t_{P}$. This transition spans an $\mathrm{X}$-axis range of $-3 / 2$. For this to define a constant at harmonic fraction of $1 / 2$, and $\mathrm{X}$-axis location of $-1 / 2$, the original mass must start at $\mathrm{X}$-axis value of 1 . The $\left[\left(v_{n^{0}}\right) s\right]^{2}$ mass must be matter in a black hole. This value has also been shown to be associated with all of the quarks so this point is of fundamental significance, and not a new observation in the HNH [26] [27] [31]. $\left[\left(v_{n^{0}}\right) s\right]^{2}$ equals 2.13456013 $(11) \times 10^{32} \mathrm{eV}$, or $3.80519890(19) \times 10^{-4} \mathrm{~kg}$. The product of $3.10077661(24) \times 10^{-36}$ and $\left[\left(v_{n^{0}}\right) s\right]^{2}$ equals the derived CMB, $v_{\mathrm{CMB}_{d}}, 160.041737(06) \times 10^{9} \mathrm{~Hz}$, Equation (16), Table 1 and Table 2. The reported experimental peak spectral radiance value is approximately $160 \times 10^{9} \mathrm{~Hz}$. The derived exponent equals $4.79707584(24) \times$ $10^{-1}$, and the $\delta_{d}$ is $-2.02924155(10) \times 10^{-2}$, Equation (16). The known experimental exponent equals approximately $4.7972 \times 10^{-1}$, and the $\delta_{k}$ is $-2.027 \times 10^{-2}$. Equations (17) and (18) is the derivation from $\beta_{\mathrm{HOd} \text {. }}$ 


$$
\begin{gathered}
v_{C M B_{d}}=v_{K E_{H_{0_{d}}}} \times\left(v_{n^{0}} s\right)^{2}=160.041737(06) \times 10^{9} \mathrm{~Hz} \\
v_{C M B_{d}}=\frac{\left(v_{n^{0}} s\right)^{2-a w k-\frac{3}{2} \times(1+a w k-b w k-b e m)}}{2}=160.041737(06) \times 10^{9} \mathrm{~Hz} \\
v_{C M B_{d}}=\frac{\left(v_{n^{0}} s\right)^{2-a w k} \times\left(\beta_{H_{0_{d}}}\right)^{2}}{2}=160.041737(06) \times 10^{9} \mathrm{~Hz}
\end{gathered}
$$

\subsection{Derivation of the Temperature from $v_{C M B_{d}}$ Using the Wien's Displacement Law}

The $v_{\max }$ for the Wien's displacement law is $58.7267923 \times 10^{9} \mathrm{~Hz}$ per K. [32] The $v_{C M B_{d}}$ divided by this Wien's constant is the temperature in Kelvin. This value is from a communication by Markus Nielbock [32]. The derived temperature is $2.72519 \mathrm{~K}$, Equation (19). The known range is $2.72548 \pm 0.00057 \mathrm{~K}$.

$$
2.72519 K_{d}=\frac{160.041737(06) \times 10^{9} \mathrm{~Hz}}{58.7267923 \times 10^{9} \frac{\mathrm{Hz}}{\mathrm{K}}}
$$

\subsection{The Inter-Changeable Inter-Relationships of $t_{P}^{2}, H_{0}$, and CMB}

The $t_{P}^{2}$ is derived from either $H_{0 d}$ or $\mathrm{CMB}_{\mathrm{d}}$ utilizing $\left[\left(v_{n^{0}}\right) s\right]$, integer fractions, and $b w k$, awk, and bem, Equation (20).

$$
t_{P_{d}}^{2}=\frac{H_{0_{d}}}{\left(v_{n^{0}} s\right)^{(128 / 35-3 / 4) \times(1+a w k-b w k-b e m)}}=\frac{2 C M B_{d}}{\left(v_{n^{n}} s\right)^{(2+(1+a w k-b w k-b e m) \times(128 / 35-3 / 2))}}=1.82617126(91) \times 10^{-86} \mathrm{~s}^{2}
$$

The $\mathrm{CMB}_{\mathrm{d}}$ is derived from either $H_{0 d}$ or $t_{P d}^{2}$ utilizing $\left[\left(v_{n^{0}}\right) s\right]$, integer fractions, and $b w k$, awk, and bem, Equation (21).

$$
v_{C M B_{d}}=\frac{H_{0_{d}}^{2} \times v_{n^{0}} s^{2}}{2 \times v_{n^{0}} s^{-a w k}}=\frac{t_{P d}^{2} \times v_{n^{0}} s^{(2+(1+a w k-b w k-b e m) \times(128 / 35-3 / 2))}}{2}=160.041737(06) \times 10^{9} \mathrm{~Hz}
$$

The $H_{0 d}$ is derived from either $v_{C M B_{d}}$ or $t_{P d}^{2}$ utilizing $\left[\left(v_{n^{0}}\right) s\right]$, integer fractions, and bwk, awk, and bem, Equation (22).

$$
H_{0_{d}}=\sqrt{\frac{2 v_{C M B_{d}}}{v_{n^{0}} S^{2+a w k}}}=t_{P d}^{2} \times\left(v_{n^{0}} S\right)^{((1+a w k-b w k-b e m) \times(128 / 35-3 / 4))}=2.2972666(11) \times 10^{-18} \mathrm{~Hz}
$$

\section{Discussion}

A robust model that scales from quantum to cosmic physical constants does not exist. The SM and ST fail. An important unique aspect of the harmonic neutron hypothesis is that it does resolve some of these limitations by analyzing the scaling relationships between the physical constants as a classic unified harmonic power law system. Derivation of an accurate CMB, and directly relating CMB to previously published derived values of $H_{0}$ and $t_{P}$ supports the validity of the model. There is no experimental astronomy data utilized. The initial data utilized for all of the calculations are subatomic data. This accounts for the high resolution result. It is remarkable that three of the most important constants related to cosmology are all related to a single $\delta$-line that defines gravity and Planck time.

In 2009 it was proposed that the fundamental constants were related to four physical values, $n^{0}, e^{-}$, $\mathrm{a}_{0}$, and $R$; and integers. Composite values uniting the subatomic properties of hydrogen including: $a w k, b w k$, and bem, were published. Only these values have been used for all of the subsequent physical constant derivations. The 
composite slopes and Y-intercepts utilized on power law plots in the interval have been shown to accurately predict and derive some of the most important physical constants. Utilization ofthis highly limited physical datain conjunction with fixed integer fractions makes it very unlikely that all of these derivations are coincidence. The predicted $H^{0}$ was $125.120961 \mathrm{GeV} / \mathrm{c}^{2}$ in 2014 , and recent data reports a mass of $125.09 \pm 0.21$ (stat.) \pm 0.11 (syst.) $\mathrm{GeV} / \mathrm{c}^{2}$ [33]. The values of the quarks were accurately predicted [31].

The HNH is based on pure integer mathematical properties. It has recently been shown that $\pi / 2$ can be derived from the orbital energy levels seen in the hydrogen atom [34]. We have shown that it is possible to the first approximation derive the properties of hydrogen from the frequency of the neutron only in conjunction with the prime numbers 2, 3, 5, 7, 11 [30]. The quarks and leptons are associated with logical progressions of prime number factors defining composite numbers of the partial fractions [29]. All of physics is speculated to be based on pure mathematical properties in the $\mathrm{HNH}$.

A high accuracy CMB is an important physical constant for physics. The experimental data are moderately precise [1]-[13]. The HNH also explains CMB's precise logical origin. The CMB in this model is not dependent on a Big Bang event. There are other interpretations [14]. The rationale in the $\mathrm{HNH}$ is identical to all quantum fundamental constants. They are related to harmonics, power laws, and exponential probabilistic mathematics. That is the only justification needed. The only possible observable fundamental constants are related integer or harmonic fraction exponents. In a quantum system that is all the justification needed for them to be observable.

In the $\mathrm{HNH} t_{P}, \mathrm{CMB}, H^{0}$, and $H_{0}$ are analogous to the other free space constants, permittivity and permeability, and represent natural inherent properties of space. Conceptually they need not be the result of some other previous physical event such as the Big Bang. Both CMB and $H_{0}$ are viewed within the standard model of astronomy as "transient" values that are measured today, but will evolve over many years. This is not the case within the HNH. There will never be any actual physical proof that these values are changing because of their immense time scale.

The HNH model is purely quantum and it is inappropriate, and conceptually inaccurate to try, and interpret physical phenomena from a classic perspective including all of the cosmology or quantum constants. There are many probabilistic properties of cosmic phenomena. The location of short gamma foci are randomly distributed. The dimension of galaxies and rotation rates of stars in galaxies are distributed over a range, but there is a general pattern.

This derivation demonstrates that $H_{0}$ has a triple physical context. $H_{0}$ is typically viewed as a measure of the expansion of the universe. In this model as a rotation rate of the visible universe, a cosmic "year". $H_{0}$ can be viewed as associated with a specific $\beta$. The kinetic energy of that $\beta^{2}$ follows the classic definition of the product of the mass times $\beta^{2}$ divided by 2 . $H_{0}$ also represents a red shift factor that is associated with a reciprocal of Lorentz factor. It is hypothesized that this Lorentz factor should be related to the maximum cosmic ray energy. The harmonic fraction is logically related to $3 / 2,1+(1 / 2)$. CMB is the smallest cosmic ray and related to a harmonic fractions of $1 / 2,1-(1 / 2)$. Therefore its harmonic fraction partner 3/2, $1+(1 / 2)$ should be the largest cosmic ray. This is analogous to the relationship of the quarks. Both of these systems have the same harmonic fraction, but just inverse signs.

$\left[\left(v_{n^{0}}\right) s\right]^{2}$ has been speculated to represent an essential physical constant. It represents the black hole domain, and is an integer exponent in the consecutive integer series of forces. It has previously been shown to be related to all of the quarks [26] [31]. There are three quark power law lines that all converge on the $(1,0)$ point which represents $\left[\left(v_{n^{0}}\right) s\right]^{2}$ on the power law plots. Therefore all of the quarks are associated with this speculated physical entity. In this case CMB represents the kinetic energy of this mass with a $\beta$ related to $H_{0}$. This is combining gravity related to $G$ and the $t_{P}^{2}$ line, and gravity related to black holes as a composite.

This is a unique unification of three of the most important cosmology observations into one unified system all based on a common line that defines Planck time. $t_{P}$ is a natural composite by definition. It is the only physical constant that spans all domains. The values of $t_{P}, H_{0}$, and $v_{C M B}$ in the existing SM are not directly mathematically inter-related variables. They are in part conceptually related, but not in a format that helps scale their relative values. This paper shows that they are all closely related, and all are related to points on the $t_{p}^{2}$ line. The fact that the known values are spaced to exactly conform to the only possibilities based on the HNH also supports the $\mathrm{HNH}$. 


\section{Conclusion}

The harmonic neutron hypothesis accurately scales quantum to cosmologic physical phenomena. This is a property of power laws. This method allows for derivation of physical constants independent of any specific associated typical experimental data. This is a classic property of all harmonic systems so this is not a new concept. The fundamental constants viewed as a unified harmonic power law system are a new concept. This is not the standard perspective of the Standard Model, but all of the foundations of the HNH are within the SM. The SM also fails to unite quantum and cosmic phenomena. The fundamental cosmologic constants CMB, $H_{0}$, and $t_{P}$ can all be derived and accurately predicted from subatomic data only. The predictions exceed the known experimental precision so they could be of experimental significance. These constants are also mathematically and conceptually closely inter-related. This is a new observation uniting cosmology within a harmonic system. These constants are all based on Planck time. Therefore all the observable cosmic phenomena including black holes are directly related to the single unifying force of gravity. It is assumed that the maximum cosmic ray energy will represent another harmonic fraction scaled by gravity as well.

\section{Acknowledgements}

I would like to thank Richard White MD, and Richard Vento for their support and help.

\section{References}

[1] Penzias, A.A. and Wilson, R.W. (1965) A Measurement of Excess Antenna Temperature at 4080 Mc/s. Astrophysical Journal, 142, 419-421. http://dx.doi.org/10.1086/148307

[2] Ellis, G.F., Poltis, R., Uzan, J.P. and Weltman, A. (2013) The Blackness of the Cosmic Microwave Background Spectrum as a Probe of the Distance-Duality Relation. http://arxiv.org/abs/1301.1312v2

[3] Mather, J.C., Cheng, E.S., Eplee, R.E., Isaacman, R.B., Meyer, S.S. and Shafer, R.A. (1990) Astrophysical Journal, 354, L37-L40. http://dx.doi.org/10.1086/185717

[4] Halverson, W. Leitch, E.M., Pryke, C., Kovac, C.J., Carlstrom, J.E., Holzapfel, W.L., Dragovan, M., Cartwright, J.K., Mason, B.S., Padin, S., Pearson, J.T., Readhead, A.C.S. and Shepherd, M.C. (2002) Astrophysical Journal, 568, No. 1. http://dx.doi.org/10.1086/338879

[5] Netterfield, C.B., Ade, P.A.R., Bock, J.J., Bond, J.R., Borrill, J., et al. (2002) The Astrophysical Journal, 571, 604. http://dx.doi.org/10.1086/340118

[6] Jaffe, A.H., et al. (2001) Physical Review Letters, 86, 3475. http://dx.doi.org/10.1103/PhysRevLett.86.3475

[7] Mangilli, S. Plaszczynski, M. and Tristram, M. (2015) MNRAS, 453, 3174-3189. http://arxiv.org/abs/1503.01347v1

[8] Planck Collaboration: Ade, P.A.R. (2015) Planck 2015 Results. XX. Constraints on Inflation, Astronomy \& Astrophysics. http://arxiv.org/abs/1502.02114

[9] Planck Collaboration: Ade, P.A.R. (2013) Planck 2013 Results. I. Overview of Products and Scientific Results. Astronomy and Astrophysics, 571.

[10] Bennett, C.L., et al. (2012) Nine-Year Wilkinson Microwave Anisotropy Probe (WMAP) Observations: Final Maps and Results. http://arxiv.org/abs/1212.5225

[11] The Planck Collaboration (2013) Planck (2013) Results. XXVII. Doppler Boosting of the CMB: Eppur si muove. http://arxiv.org/abs/1303.5087

[12] Scott, D. (2005) The Standard Cosmological Model. http://arxiv.org/abs/astro-ph/0510731

[13] Naselsky, P.D., Novikov, D.I. and Novikov, I.D. (2006) The Physics of the Cosmic Microwave Background. Cambridge University Press, New York. http://dx.doi.org/10.1017/CBO9780511536373

[14] Alia, A.F. and Dasc, S. (2015) Physics Letters B, 741, 276-279.

[15] Lykken, J. and Spiropulu, M. (2014) Scientific American, 310, 34-39. http://dx.doi.org/10.1038/scientificamerican0514-34

[16] Basilakos, S., Dasb, S. and Vagenasa, E.C. (2010) Journal of Cosmology and Astroparticle Physics, 27, 1-9. http://iopscience.iop.org/article/10.1088/1475-7516/2010/09/027/meta

[17] Lipovka, A. (2014) Journal of Applied Mathematics and Physics, 2, 61-71.

[18] Bonamente, M., Joy, M.K., Laroque, S.J., Carlstrom, J.E., Reese, E.D. and Dawson, K.S. (2006) The Astrophysical Journal, 647, 25-54. http://dx.doi.org/10.1086/505291 
[19] Freedman, W.L. and Madore, B.F. (2010) Annual Review of Astronomy and Astrophysics, 48, 673-710. http://dx.doi.org/10.1146/annurev-astro-082708-101829

[20] Bennett, C.L., Larson, D., Weiland, J.L., Jarosik, N., Hinshaw, G., Odegard, N., Smith, R.S., Hill, K.M., Gold, B., et al. (2013) The Astrophysical Journal Supplement Series, 208, 19. http://dx.doi.org/10.1088/0067-0049/208/2/20

[21] Freedman, W.L., Madore, B.F., Scowcroft, V., Burns, C., Monson, A., Persson, S.E., Seibert, M. and Rigby, J. (2012) The Astrophysical Journal, 758, 24. http://dx.doi.org/10.1088/0004-637X/758/1/24

[22] Ade, P.A.R., Aghanim, N., Armitage-Caplan, C., Arnaud, M., Ashdown, M., Atrio-Barandela, F., et al. (2014) Astronomy \& Astrophysics, 571, 16.

[23] Chakeres, D.W. (2009) Particle Physics Insights, 2, 1-20.

[24] Chakeres, D.W. (2011) Particle Physics Insights, 4, 19-23. http://dx.doi.org/10.4137/PPI.S7961

[25] Chakeres, D.W. (2011) Particle Physics Insights, 4, 25-31. http://dx.doi.org/10.4137/PPI.S8241

[26] Chakeres, D.W. (2013) Particle Physics Insights, 6, 1-7. http://dx.doi.org/10.4137/PPI.S12390

[27] Chakeres, D.W. (2014) Journal of Modern Physics, 5, 1670-1683. http://dx.doi.org/10.4236/jmp.2014.516167

[28] Chakeres, D.W. and Vento, R. (2015) Journal of Modern Physics, 6, 283-302. http://dx.doi.org/10.4236/jmp.2015.63033

[29] Chakeres, D.W. and Vento, R. (2015) Advances in Pure Mathematics, 5, 240-250. http://dx.doi.org/10.4236/apm.2015.55025

[30] Chakeres, D.W. and Vento, R. (2015) Journal of Modern Physics, 6, 2145-2157. http://dx.doi.org/10.4236/jmp.2015.614218

[31] Chakeres, D.W. and Vento, R. (2016) Journal of Modern Physics, 7, 145-155. http://dx.doi.org/10.4236/jmp.2016.71016

[32] Balog, Z., Muller, T., Nielbock, M., Altieri, B., Klaas, U., Blommaert, J., Linz, H., Lutz, D., Moor, A., Billot, N., Sauvage, M. and Okumura, K. (2014) Experimental Astronomy, 37, 129-160. http://dx.doi.org/10.1007/s10686-013-9352-3

[33] Aad, G., et al. (ATLAS, CMS Collaborations) (2015) Physical Review Letters, 114, Article ID: 191803. http://dx.doi.org/10.1103/PhysRevLett.114.191803

[34] Friedmann, T. and Hagen, C.R. (2015) Journal of Mathematical Physics, 56, Article ID: 112101. http://dx.doi.org/10.1063/1.4930800 